\title{
Exploring service-oriented system engineering challenges: a systematic literature review
}

\author{
Qing Gu • Patricia Lago
}

Received: 3 November 2008 / Revised: 8 June 2009 / Accepted: 18 June 2009 / Published online: 15 July 2009

(C) The Author(s) 2009. This article is published with open access at Springerlink.com

\begin{abstract}
Service-oriented system engineering (SOSE) has drawn increasing attention since service-oriented computing was introduced in the beginning of this decade. A large number of SOSE challenges that call for special software engineering efforts have been proposed in the research community. Our goal is to gain insight into the current status of SOSE research issues as published to date. To this end, we conducted a systematic literature review exploring SOSE challenges that have been claimed between January 2000 and July 2008. This paper presents the results of the systematic review as well as the empirical research method we followed. In this review, of the 729 publications that have been examined, 51 were selected as primary studies, from which more than 400 SOSE challenges were elicited. By applying qualitative data analysis methods to the extracted data from the review, we proved our hypotheses about the classification scheme. We are able to conclude that the SOSE challenges can be classified along two dimensions: (a) based on themes (or topics) that they cover and (b) based on characteristics (or types) that they reveal. By analyzing the distribution of the SOSE challenges on the topics and types in the years 20002008, we are able to point out the trend in SOSE research activities. The findings of this review further provide empirical evidence for establishing future SOSE research agendas.
\end{abstract}

Keywords Service-oriented system engineering . SOSE challenge $\cdot$ Empirical software engineering . Systematic review

\section{Introduction}

Service-oriented system engineering (SOSE) [1] addresses systematic, disciplined and quantifiable approaches to develop service-oriented systems. A common concept shared among these approaches is software being used as a service for consumption. Web services [2], grid and cloud computing [3], SaaS [4] and Web 2.0 [5] are some emerging trends that adopt this concept. As a relatively new field, numerous SOSE challenges have been claimed in the SOSE research community. In this paper, we define SOSE challenges as SOSE-related problems or issues that demand research efforts.

Facing a large number of SOSE challenges, Kontogiannis et al. [6,7] and Papazoglou [8] pointed out that a well focused research agenda is necessary to consolidate and stream current SOSE research efforts. Currently, several workshops, conferences, projects and initiatives are dedicated to establish SOSE research agenda, such as the International Workshop on Systems Development in SOA Environments (SDSOA) and the Networked European Software and Services Initiative (NESSI). In addition, conferences, workshops, and journals possibly contributing to such SOSE research agenda but having a broader focus include ICSOC $^{1}$ in 2003, SCC ${ }^{2}$ and

Q. Gu $(\bowtie) \cdot$ P. Lago

Department of Computer Science, VU University,

Amsterdam, The Netherlands

e-mail: qgu@few.vu.nl

\footnotetext{
${ }^{1}$ International Conference on Service Oriented Computing.

${ }^{2}$ International Conference on Services Computing.
} 


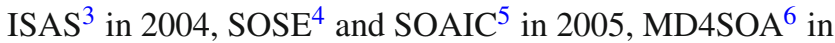
2006, SOCA $^{7}$ in 2007 and IESS $^{8}$ in 2010.

The commonality among these research activities is that they are all centered around a subset of SOSE challenges rather than a complete set, depending upon their research focuses. For instance, the SOA research agenda proposed by Kontogiannis et al. [7] discusses research issues related to SOA adoption for organizations. The challenges raised due to the collaboration between multiple parties in serviceoriented development [9], for example, are not covered in this agenda. As another example, the SOC research roadmap proposed by Papazoglou et al. [8] presents research issues in terms of extended SOA, whose core layers are service foundations, service compositions, and service management. Cross-cutting concerns, such as SOA education [10], are not considered in this roadmap.

The aforementioned research agenda and roadmap present a list of important challenges for service-oriented systems to reach their full potential. Without a holistic view on "all" the SOSE challenges posed by researchers, less well-known challenges are likely to be ignored. An overview of a complete set of SOSE challenges can provide a firm basis for establishing SOSE research agenda.

Brereton et al. [11] are the pioneers who conducted a systematic review of issues that need to be addressed in engineering service-oriented systems. The results of their review include a number of issues vital for engineering serviceoriented systems, for which solutions as well as associated research methods have been investigated. However, it has the following limitations.

First of all, the review conducted by Brereton et al. restricted the data sources to journals only. We understand that journal papers usually guarantee the quality of the studies due to strict review procedures, which is the main reason why six journals that are strongly related to software engineering research were chosen as primary sources. Conference and workshop proceedings (major sources for researchers to discuss open issues, immature ideas and research challenges), however, were not considered in the review. Second, informal data analysis methods have been used for data synthesis. The identified issues have been grouped, but no synthesis method has been described. Third, a framework that was designed for classifying component-based software engineering (CBSE) issues was adopted for classifying the identified SOSE issues

\footnotetext{
${ }^{3}$ International Service Availability Symposium.

${ }^{4}$ International Workshop on SOSE.

${ }^{5}$ International Workshop on Service-oriented Applications, Integration and Collaboration.

${ }^{6}$ Modeling, Design, and Analysis for Service-oriented Architecture Workshop.

7 Journal of Service Oriented Computing and Applications.

${ }^{8}$ International Conference on Exploring Services Sciences.
}

as well. Although this framework is applicable to classify SOSE issues due to similarities between SOSE and CBSE, it provides a limited classification approach because the framework was pre-defined rather than emerged from the identified SOSE issues.

Last, the studies that have been analyzed in Brereton et al.'s review were published between 2000 and June 2004. After the establishment of all the aforementioned conferences/ workshops and journals on service-oriented computing, it is time to perform an up-to-date systematic review of SOSE issues or challenges.

A systematic literature review (also called systematic review) is an evidence-based approach to thoroughly search studies relevant to some pre-defined research questions and critically select, appraise, and synthesize findings to answer such research questions. Unlike traditional narrative reviews, systematic reviews often include focused research questions, explicit search strategies, explicit selection criteria, qualitative as well as quantitative-summary. Because systematic reviews methodologically encourage rigorous review results, we decided to perform a systematic review of all the SOSE challenges that have been proposed so far.

This paper reports on the results of our systematic review aiming at identifying and classifying SOSE challenges. In doing that, we provide an overview of the-state-of-the-art of SOSE challenges, and a key for reading and interpreting them. In addition, this paper presents a number of interesting findings, including SOSE emergence laws, a parallel between the SOSE challenges cycle and the Gartner Hype Cycle, and the importance of inter-relationships between SOSE challenges. Our findings also ring a bell to the research community that data-related SOSE issues require more attention in the SOSE research community.

As suggested in [12], we organize the content of this paper according to the structure proposed in [13]. In Sect. 2, we describe the process of our systematic review, including the need for a systematic review, the definition of the research questions, the description of search and selection strategy, the explanation of data extraction forms, and the introduction of data synthesis methods. The description of the process defines the review protocol. Section 3 presents the included studies. Our answers to the research questions are presented in Sect. 4. In Sect. 5 we mainly discuss the threats to validity. Finally, we conclude the paper in Sect. 6 and discuss directions for further research.

\section{Research method}

\subsection{Systematic review}

Systematic reviews are particularly powerful in collecting and analyzing existing work, which is a common task in 
establishing background knowledge in any research. The main reasons to perform systematic reviews, outlined in [12], include (1) summarizing the existing evidence about a specific software engineering issue; (2) identifying gaps in current research to suggest further research directions, and (3) positioning new research activities in a research framework.

We chose systematic review as our research method due to its methodological advantages and applicability to our research questions. From the methodological point of view, systematic reviews are methodical. They maximize the chance to retrieve complete data sets and minimize the chance of bias. From the applicability point of view, our research questions are in accordance with the first reason summarized in [12], i.e., summarizing the existing evidence.

To conduct the systematic review, we followed the guidelines suggested in [12] for performing systematic reviews in software engineering. According to the guidelines, a systematic review constitutes three main phases, namely (1) planning the review, (2) conducting the review, and (3) reporting the review. The main activities in the planning phase are to specify research questions and develop a review protocol. A review protocol is of critical importance to a systematic review because it specifies search, selection, data extraction, and synthesis strategies. After having specified and evaluated the protocol, its execution is the main activity in the conducting phase. Finally, the results of the review should be reported.

\subsection{Research questions}

SOSE challenges have been often addressed in different studies from different perspectives simultaneously, making challenges appear fragmented. For instance, one challenge can be addressed from the product perspective in one study and the process perspective from another study. This challenge therefore assumes a different meaning in each of these two studies. To give an example, service design [8] is one of the SOSE challenges. When addressed from the product perspective, it concerns the quality (e.g., reusability [14]) and characteristics (e.g., loose coupling, implementation neutrality [15]) of resulting services. When addressed from the process perspective, it concerns the way service design is carried out (e.g., collaborative design [9]) and its supporting tasks (e.g., decide the level of coupling in service design [16]). From this example, we can see that all these challenges (quality, characteristics, approaches, and tasks) are aspects of service design. If we were able to group or classify inter-related challenges, research efforts could be better focused to address all aspects of a clustering (super-)challenge.

SOSE challenges are also described in different studies at various levels of abstraction, making challenges appear fictively independent or isolated. For instance, typically, challenges described at a high level (or conceptual level) are about proposing requirements for engineering activities or resulting products, including architectures or components of service-oriented systems; challenges described at a lower level (or implementation level) often advocate the need for new techniques or tools that can be used in service-oriented engineering activities. The implementation-level challenges are often the "solutions" towards the conceptual level challenges. To give an example, a SOSE challenge claiming that services should be dynamically discoverable is a conceptual level challenge because it does not directly address implementation details. An implementation-level challenge could be the need for techniques to specify services not only in syntax but also in semantics because specifying services' semantics is a way to enable service discovery. We can see that the challenge dynamically discoverable services is dependent on the challenge specifying services. If we are able to link the challenges that are inter-dependent, the direction of research efforts can be more precise.

In order to explore the links between SOSE challenges, in our early work [17] we proposed a framework aiming at classifying SOSE challenges and explicitly expressing the inter-dependencies between them. In that work, we realized that without a holistic view of the existing SOSE challenges, a sound framework is very hard to create. We also realized that a classification scheme for SOSE challenges is essential for such a framework due to the diverse nature of SOSE challenges. With this motivation in mind, the aim of this systematic review is not only to identify all the claimed SOSE challenges, but also to classify them so that inter-related and inter-dependent challenges can be grouped. Before conducting this systematic review, we defined two hypotheses on the classification of SOSE challenges.

- Hypothesis 1: SOSE challenges can be classified based on their topics.

We formulated this hypothesis because SOSE challenges are addressed at different levels of detail. Some implementation-level challenges can be regarded as sub-challenges of a conceptual-level challenge. We may consider all these challenges as illustrating the same topic.

We define topic as the subject or theme of a challenge. Challenges illustrating the same topic should address the same subject but with a different focus. For instance, dynamic service discovery and semantically enhanced service discovery are both about service discovery. The former focuses on performing service discovery in a dynamic manner rather than a static manner, while the latter emphasizes enhancing the way that services are discovered by adding semantic annotations to service specifications.

The conditions for this hypothesis include (1) each challenge should have one and only one topic; (2) the 
abstraction level of a challenge should be lower or equal to the abstraction level of its topic.

- Hypothesis 2: SOSE challenges can be classified based on their types.

We formulated this hypothesis because SOSE challenges are addressed from different perspectives, such as process, product, and architectural perspectives. We may regard all the challenges that are addressed from the same perspective as one type.

We define type as a general form or style common to a number of challenges. The challenges that are of the same type must have distinguishing characteristics in common. For instance, composability analysis, transaction management tools, and encryption are three SOSE challenges. These challenges are common methods or tools that can be used to accomplish some tasks: composability analysis is used in service composition, transaction management tools support transaction management, and encryption is a way to improve security. Therefore, these three challenges can be regarded as of the same (general) type, e.g., technique challenges.

The condition for this hypothesis is that each challenge should have one and only one type.

With these two hypotheses in mind, we specify the following research questions:

- Q1: What SOSE challenges have been claimed in the research community so far?

- Q2: How to classify the claimed SOSE challenges?

- Q2.a: What are the characteristics of the challenges? Can we classify these challenges by types based on their characteristics?

- Q2.b: What are the topics addressed by the challenges? Can we summarize a list of topics that these challenges have covered?

- Q2.c: Are there any other ways to classify SOSE challenges?

\subsection{Review protocol}

A review protocol is a plan specified prior to the execution of a systematic review. This plan describes how to search and select relevant studies as well as how to analyze extracted data to answer pre-defined research questions. A well-defined review protocol is essential to a systematic review since it encourages less researcher bias [12].

The main components of the review protocol include data sources, search strategy, study selection strategy, data extraction method, and data synthesis. The first three components define the scope of the study and explain the motivation behind it. The last two components describe how the results are concluded. All these components are essential for the readers to fully understand and appraise the review and critical for the researchers in the same domain to replicate the review or repeat the review with other datasets [18]. We, therefore, explicitly describe the review protocol in this subsection.

\subsubsection{Data sources}

The following electronic libraries give a reasonable confidence of covering all relevant publications. Therefore, we use these libraries as our main resources:

- IEEE Explore

- ACM Digital Library

- ISI Web of Knowledge

- SpringerLink

- ScienceDirect

- Wiley Inter Science Journal Finder

\subsubsection{Search strategy}

The research questions listed in Sect. 2.2 require the same set of data collected from the literature. Research question Q1 is relatively more straightforward and question $\mathrm{Q} 2$ requires further data synthesis. The search strategy was therefore developed based on research question Q1.

Question Q1 contains two key terms, namely: SOSE and challenge. A list of related terms was constructed for each of these two terms, as shown in Table 1.

A search string was then constructed using Boolean "and" to connect the two key terms and "or" to allow synonyms.

(SOSE or SOA or "service-oriented" or "service computing" or "service-based" or "service-centric" or "service engineering") and (challenge or approach or agenda or roadmap or "road map" or "overarching concern" or "research issue")

Besides the search string, the range of study dates should also be defined in the search strategy. For instance, Brereton et al. [11] have chosen 2000 as the starting year for their review because SOAP was first submitted to W3C in 2000

Table 1 Related terms for the two key terms: SOSE and challenge

\begin{tabular}{ll}
\hline SOSE & Challenge \\
\hline SOA & Approach \\
Service-oriented & Agenda \\
Service computing & Roadmap \\
Service-based & Road map \\
Service-centric & Overarching concern \\
Service engineering & Research issue
\end{tabular}


and web services could be implemented from then on. Although major conferences/workshops on service-oriented systems like ICSOC started in 2003, we decided to use 2000 as the start date to eliminate any chance to overlook SOSE challenges proposed in conferences/workshops or journals which do not specifically focus on service-oriented systems.

The last decision we made was to apply search queries to titles, abstracts, or full-text of publications. In our experience titles do not always correctly or completely reflect the content of publications. Searches based on titles cannot provide us with a reasonably complete set of publications relevant to our research questions. On other hand, if we search in fulltext, the chance of finding irrelevant publications is too high. Abstracts usually provide brief and moderate summaries of publications. We, therefore, decided to apply search queries to the abstracts of the studies. This means a study is selected as a candidate study if its abstract contains the keywords defined in the search string.

Because different digital search engines often provide different search interfaces and require different search syntaxes, we had to adapt the search string for each search engine.

\subsubsection{Study selection}

Some of the studies might contain the keywords used in the search string but are irrelevant to our research questions. Study selection therefore has to be performed to include only studies that contain useful information for answering the research questions. The selected studies constitute primary studies for a systematic review.

Accordingly, a set of inclusion and exclusion criteria are specified based on the scope of the review and the quality of the studies. A study is selected as a primary study if it satisfies all the pre-defined inclusion criteria and is eliminated if it fulfills any of the pre-defined exclusion criteria. These criteria, together with the corresponding motivation and rationale are given in Table 2.

Inclusion criterion I1 limits our review to studies that are strongly related to SOSE. More specifically, exclusion criteria $\mathrm{E} 1$ and $\mathrm{E} 2$ are used to eliminate studies that are irrelevant and marginally related to SOSE, respectively, and E3 indicates that SOSE in a specific application domain is beyond the range of our review.

Inclusion criterion I2 limits our review to studies that are strongly related to challenges. More specifically, exclusion criterion E4 indicates that solutions to SOSE challenges are beyond the scope of this review; and E5 points out that a study that is strongly related to SOSE but does not propose SOSE challenges is not relevant in our review.

The last criteria I3 and E6 are used to select studies that reach the quality of scientific papers. In our future work, we plan to conduct another systematic review which focuses on the studies that are presented in forms other than scientific papers.

\subsubsection{Data extraction}

The reference details of each primary study are recorded using Endnote (http://www.endnote.com). For quantitative analysis purposes, we also record the type of paper (conference/workshop or Journal/Book chapter) and the year of publications in a spreadsheet.

Each primary study is analyzed concentrating on identifying SOSE challenges. When a study describes a challenge regarding SOSE or proposes a SOSE research issue, we elicit SOSE challenges from this study in such a way that each SOSE challenge is the smallest unit (i.e. cannot be decomposed to multiple challenges from its original description). For instance, suppose a study describes a SOSE research issue as "services should be discoverable and composable", we elicit two SOSE challenges as "services should be discoverable" and "services should be composable". In this way, each elicited SOSE challenge has one focus.

All the identified challenges are documented in a spreadsheet in terms of their names, descriptions, and rationale (if present). The data model for the data extraction form is presented in Fig. 1.

\subsubsection{Data synthesis}

After all the SOSE challenges are identified from the primary studies and recorded in a spreadsheet, we have a pool of challenges in the form of descriptive text. In order to answer the question Q2 (how to classify SOSE challenges), we need to synthesize the extracted data as follows:

Classify the SOSE challenges by their topics. Reciprocal translation and line of argument synthesis, two strategies of Meta-ethnography, especially useful for interpretive reviews, were first introduced by Noblit and Hare [19].

As Kitchenham [12] suggested, reciprocal translation can be used "when researchers need to provide an additive summary on the studies that are about similar things by translating each case into each of the other cases". Instead, line of argument synthesis can be used "when researchers are concerned about what they can infer about a topic as a whole from a set of selective studies that look at a part of the issue".

In our systematic review, if some of the SOSE challenges are about a similar topic, reciprocal translation is applicable to translate each topic into each of the other topics. For instance, suppose we have identified three challenges, namely (1) dynamic service composition, (2) compose services at runtime, and (3) automatic service composition. By applying reciprocal translation, we may conclude that all these 
Table 2 Motivation and rationale for inclusion and exclusion criteria

\begin{tabular}{ll}
\hline Inclusion criteria & Exclusion criteria (exceptions to inclusion criteria)
\end{tabular}

I1 A study that is mainly about service-oriented systems or service-oriented architectures.

Motivation: we are interested in challenges that are related to SOSE. This implies that studies that are about SOA or service-oriented systems are relevant to our research questions

I2 One of the main objectives of a study is to present service engineering research challenges, issues, open questions, etc. Motivation: if one of the objectives of a study is to propose a challenge, we expect that this challenge has not been proposed before.

I3 A study that is in form of a scientific paper.

Motivation: a scientific paper guarantees a certain level of quality and contains reasonable amount of content.
E1 A study that is not about service-oriented architecture. Rationale: since we use SOA as one of the keywords in the search strings, studies that have "SOA" in their abstracts but are completely irrelevant to service-oriented systems should be excluded. For instance, studies about Society Of Actuaries or Silicon-On-Anything will be excluded.

E2 A study which is marginally related to service-oriented systems.

Rationale: if the core of a study is about another field than service engineering and is only marginally related to service-oriented systems should be excluded. For instance, a study that is mainly about how to design and develop health information systems should be excluded.

E3 A study that is about SOA application in a specific domain.

Rationale: we are interested in SOSE-related challenges in general but not in specific application domains. The challenges that are very important to one application domain might be less relevant to another application domain. Although domain challenges are also vital to the application of service-orientation, it is not the focus of this study. For instance, a study proposing SOSE challenges that the military domain faces should not be included because these challenges might not be relevant or important to other application domains; however, a study proposes SOSE challenges in general and further elaborates how these challenges influence the military information system will be included.

E4 The objective of a study is only to present solutions to a SOSE challenge.

Rationale: if presenting SOSE challenges is not one of the objectives of a study, most likely the challenges (if ever mentioned) are known challenges. These known challenges should be (originally) claimed by other studies.

E5 A study that has other objective than proposing challenges or solutions.

Rationale: a study that does not propose any SOSE challenges has no value to our research questions.

E6 A study that is not in form of a scientific paper. For instance, tutorials, keynotes, presentations do not provide sufficient information.

Rationale: a study that is not a scientific paper might not have sufficient level of quality or does not provide reasonable amount of information. three challenges are about composing services without human intervention. Therefore, we may unify these three challenges by naming all of them dynamic service composition. We should apply reciprocal translation to all the identified SOSE challenges until no challenge can be translated into another.

After applying reciprocal translation to all the challenges, we examine whether one challenge is a sub-challenge of another. We use line of argument synthesis to determine the main theme of a set of challenges. For instance, we have identified the following challenges: (1) dynamic service composition, (2) integrate transaction management into the service compositions, (3) service composition language, and (4) composability analysis. All these four challenges fall under the umbrella of another challenge "service composition". We may therefore conclude that these four challenges all address the topic "service composition". In this case, "service composition" is both the name of the SOSE challenge and of the topic.

In practice, we should apply line of argument synthesis to all the challenges in an iterative manner. In the first iteration, temporary topics should be determined for all the SOSE challenges. Afterwards, we should apply line of argument synthesis to all the temporary topics in case some of the temporary topics can be further translated. We have to repeat this process until each topic is distinguishing and none of the topic is a sub-topic of another. In the end, we generate a list of topics of the SOSE challenges. 


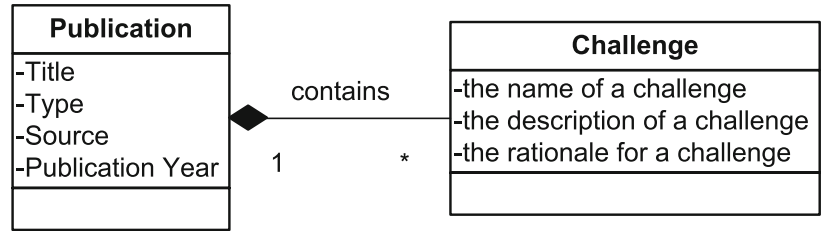

Fig. 1 The data model for data extraction forms

Classify the SOSE challenges by their types. A type of the SOSE challenges can be regarded as a term that insightfully describes the characteristics of SOSE challenges. The SOSE challenges that are assigned by the same type should share the same characteristics. A common method that is used for this purpose is the grounded theory method because the theories (types) are "grounded" in the data (the SOSE challenges) [20].

Constant comparison method, one of the grounded theory techniques, has been often used in analyzing data and generating categories of data. Although constant comparison method can be used on any set of data, it is particularly suitable for the data that are context sensitive [21] (i.e., data can be interpreted differently in different contexts).

To interpret an SOSE challenge correctly, one often needs to understand in which context the challenge is proposed and how it is addressed. For instance, Zhu [14] claims that "to make services reusable" is a challenge. Without understanding the context of this challenge, we cannot conclude whether this challenge is about designing reusable services or about what can be reused in services. By reading the context, we understand that this challenge is about the understandability of services, the price/performance ratio of services, etc. We can therefore conclude that this challenge was addressed from the quality perspective (reusability) rather than the design principle perspective.

Miles and Huberman [22] described the procedure for the constant comparison method as: coding, pattern coding, memo, refining proposition. Following this procedure, we have to label each identified SOSE challenge with emergent codes; look for patterns or trends in the codes; compare and study coded passages; record any findings in memos; and eventually, propositions can be established and refined from the memos. The iteration of these steps continues until no new proposition emerges and no modification to proposition is needed, which means theoretical saturation is reached. These propositions reflect the patterns and characteristics (types) recognized among a subset of SOSE challenges. In this way, SOSE challenges are classified by their types.

\section{Overview of the included studies}

In total, we found 729 publications, whose abstracts contain the keywords defined in the search string. By carefully eval-

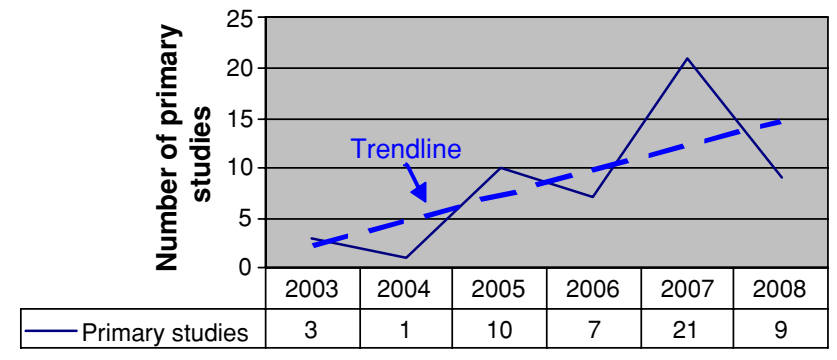

Fig. 2 Distribution of primary studies published by year

uating these publications against the inclusion and exclusion criteria, we selected 51 publications that are strongly relevant to our research questions, identified as primary studies.

Figure 2 shows the distribution of primary studies published by year as well as the trend-line. The positive trend-line slope indicates an increasing amount of research work being dedicated to identifying, proposing, and collecting SOSE challenges.

According to the results of our review, the first three primary studies proposing SOSE challenges were published in 2003 (when ICSOC was launched). Although the number of primary studies slightly drops in 2004, it tremendously increases and reaches its first peak in 2005; it slightly decreases again in 2006 followed by the second peak in 2007, which is doubled as compared to the first peak. We interpret these two drops as shifting research efforts from identifying challenges to investigating solutions. As soon as more experiences have been accumulated in engineering serviceoriented systems, researchers are keen to investigate ways to maximize the potential benefits that SOA promised, such as flexibility, agility, and reusability.

It is very interesting to notice that our interpretation on the distribution of primary studies resembles the two most prominent laws of software evolution suggested by Lehman [23]: continuous changes and increased complexity. These two laws indicate that when software is evolving, changes to software are inevitable. Maintenance efforts therefore have to be devoted to reduce the increased complexity caused by the changes. When we look at SOSE challenges, there are continuous expectations instead of continuous changes; and there are growing understandability instead of increased complexity. Consequently, the higher the expectations, the more work is dedicated to identifying challenges; the better the challenges are understood, the more work is dedicated to overcome the challenges. These two regular patterns are called SOSE emergence laws in our review.

These two SOSE emergence laws are also revealed by the summary of primary studies in terms of sources and year, as presented in Fig. 3. The sources of the primary studies include conference, workshop and symposium proceedings, as well as journal articles, and book chapters. Generally speaking, publications in journals and books represent more mature 
Fig. 3 Summary of primary studies in terms of source and year

\begin{tabular}{|l|rrrrrr|r|}
\hline Source & $\mathbf{2 0 0 3}$ & $\mathbf{2 0 0 4}$ & $\mathbf{2 0 0 5}$ & $\mathbf{2 0 0 6}$ & $\mathbf{2 0 0 7}$ & $\mathbf{2 0 0 8}$ & Grand Total \\
\hline book chapter & 0 & 0 & 2 & 0 & 4 & 0 & 6 \\
Journal & 0 & 0 & 1 & 4 & 4 & 3 & $\mathbf{1 2}$ \\
SubTotal & $\mathbf{0}$ & $\mathbf{0}$ & $\mathbf{3}$ & $\mathbf{4}$ & $\mathbf{8}$ & $\mathbf{3}$ & $\mathbf{1 8}$ \\
\hline conference & 1 & 0 & 4 & 1 & 10 & 2 & $\mathbf{1 8}$ \\
Symposium & 0 & 0 & 2 & 1 & 0 & 0 & $\mathbf{3}$ \\
Workshop & 2 & 1 & 1 & 1 & 3 & 4 & $\mathbf{1 2}$ \\
SubTotal & $\mathbf{3}$ & $\mathbf{1}$ & $\mathbf{7}$ & $\mathbf{3}$ & $\mathbf{1 3}$ & $\mathbf{6}$ & $\mathbf{3 3}$ \\
\hline Grand Total & $\mathbf{3}$ & $\mathbf{1}$ & $\mathbf{1 0}$ & $\mathbf{7}$ & $\mathbf{2 1}$ & $\mathbf{9}$ & $\mathbf{5 1}$ \\
\hline
\end{tabular}

research results than those in conference, workshop, or symposium proceedings. According to our review, the SOSE challenges elicited from journal articles or book chapters were first posed in 2005. The increasing number of SOSE challenges appearing in journal articles or book chapters in 2006 and 2007 not only indicates an incremental insight in the field, but also points out that SOSE challenges are attracting growing attention in the research community.

\section{Results of the systematic review}

\subsection{RQ1—claimed SOSE challenges}

A total of 413 SOSE challenges were elicited from the selected 51 primary studies. The distribution of the number of SOSE challenges proposed by year is illustrated in Fig. 4. While Fig. 2 is about the amount of work dedicated to identifying SOSE challenges, this distribution presents the number of SOSE challenges actually being identified. It would be reasonable to expect an increasing number of challenges with the increase in the number of publications related to challenges. Nonetheless, e.g., certain publications might identify many more challenges than others. In other words, the distribution of challenges is not necessarily the same or similar to the distribution of primary studies. For instance, we might encounter a situation in which the studies in 20032005 propose say 20 challenges, and the studies after 2005 propose only one challenge. In this hypothetical situation, the trend-line slope of SOSE challenges would be negative. The similarity between these two distributions (in Figs. 2, 4) points out that the actual results from research publications on SOSE challenges also reflect the SOSE emergence laws.

It is interesting to note that the distribution line presented in Fig. 4 reveals what we call the SOSE challenges cycle (see the dashed line). This cycle recalls the Gartner Hype Cycle [24] (both illustrated in Fig. 5). While the Gartner Hype Cycle (thicker line in the figure) graphically presents the maturity level of the application of specific technologies, the SOSE challenges cycle presents the awareness of SOSE-related research issues. These two cycles are mutually related. When SOSE faces peak of inflated expectations (see the peak of the Gartner hype cycle in Fig. 5), researchers are ambitious to investigate how to fulfill those expectations and

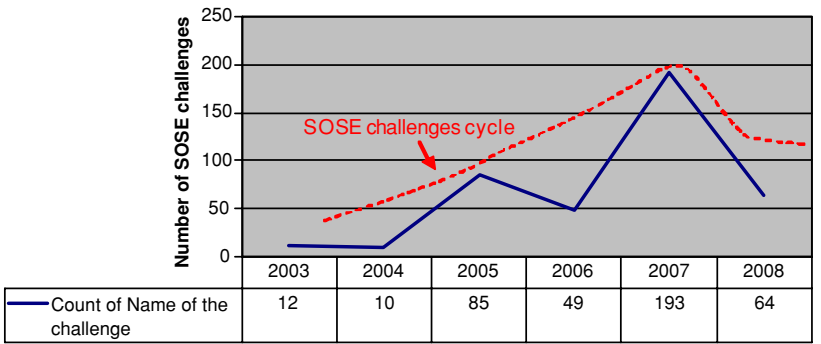

Fig. 4 Distribution of the number of SOSE challenges proposed by year

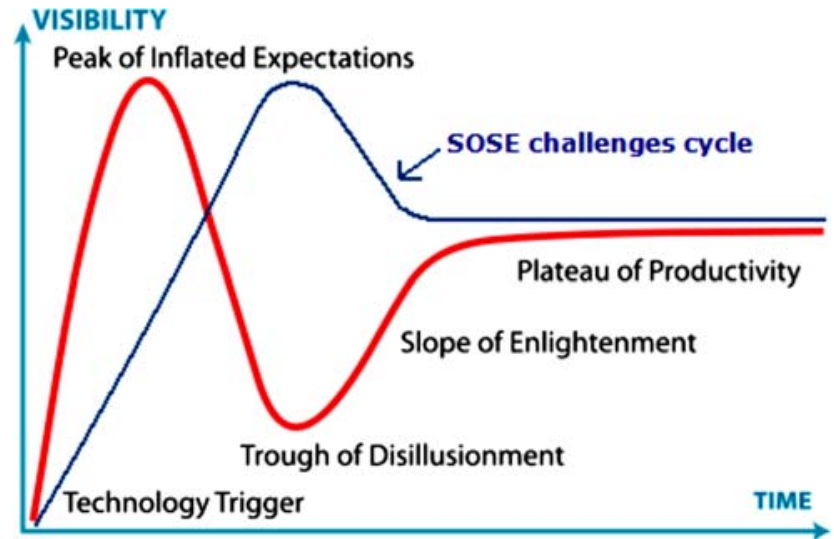

Fig. 5 Gartner Inc.'s hype cycle (Source: http://en.wikipedia.org/ wiki/Hype_cycle) and the SOSE challenges cycle

therefore the awareness of SOSE challenges tremendously increases. When investigation results start to appear, new research issues keep emerging and known research issues are further decomposed. Therefore, awareness of SOSE challenges increases further until it reaches its peak (see the peak of the SOSE challenges cycle in Fig. 5). At the same time, SOSE faces trough of disillusionment (see the base of the Gartner hype cycle in Fig. 5) since researchers are aware that there are too many SOSE challenges to be solved before SOSE reaches the level of maturity to be widely adopted.

We observe that (a) in 2007 a large number (193) of SOSE challenges have been published and (b) it is unlikely that there will be more SOSE challenges published in 2008 than in 2007 since there are only 64 challenges published before July 2008 (i.e. about one third of the challenges in 2007). Further, researchers have already accumulated knowledge 
of SOSE-related research issues from 2003 and these issues cover a wide range of SOSE research topics. Based on these observations, we deduce that the number of SOSE challenges is reaching stability (see the decreasing part of the SOSE challenges cycle in Fig. 5). We therefore argue that we are currently in the slope of enlightenment of the Gartner Hype Cycle.

\subsection{RQ2.1-topics of SOSE challenges}

By using the reciprocal translation and line of argument synthesis methods explained in Sect.2.3.5, we determined one topic for each elicited SOSE challenge and we summarized 45 topics in total. In this way, the SOSE challenges can be classified by their topics, which confirmed our first hypothesis. The summarized topics are presented in Appendix A, sorted by the descending number of challenges under each topic.

Looking at the summarized topics, we noticed that many topics are not at the same abstraction level. Some of the topics (e.g., SOA education) are broader than the others (e.g., fault handling, transaction). The different abstraction levels are caused by the fact that the topics that have been addressed as SOSE challenges elicited from the primary studies already belong to different abstraction levels. And this becomes apparent after using the synthesis methods (explained in Sect. 2.3.5) as well.

To get an overall idea about the coverage of these topics, we compared our results with the issues identified in the systematic review conducted by Brereton et al. [11], which to our knowledge is the only systematic review performed on SOSE studies. Of the 20 issues concluded in [11], 2 issues are not touched at all in our review ('payment' and 'time to market'), and 2 issues partially overlap as being only indirectly related to the topics in our review ('service replication' and 'statefulness in the context of replication'). Service replication is one of the approaches to improve the capability of fault tolerance or fault handling. In our review, although we do not find any SOSE challenge directly linked to service replication, we do find some challenges addressing fault handling. Therefore, we regard these two issues as indirectly related. The remaining 16 issues match with the topics summarized in our review.

We have to keep in mind that the publication duration covered by these two reviews is different. The review conducted by Brereton et al. covers the studies that are published between January 2000 and June 2004, whereas our review covers studies that are published between January 2000 and July 2008. Furthermore, the research questions addressed by these two reviews overlap but with a different focus: Brereton et al. analyzed the issues that occurred in engineering service-oriented systems as well as solutions proposed to address these issues, whereas our review does not investigate solutions. Therefore, the identified SOSE research top- ics between these two reviews are most likely to overlap with some differences. We therefore use the comparison results for reference purposes only, rather than validation purposes.

\subsubsection{Top three topics: quality, service, and data}

Looking at the summarized topics in Appendix A, some of them are well-known in that we have seen numerous research papers relating to those topics, but some of them are new to us in that we do not know how much research efforts have been dedicated to those challenges. To gain insight into the heaviest or lightest published research areas, we specifically zoomed into the topics that cover the highest number of challenges as well as the lowest ones.

As we can see in Appendix A, the topic of 'quality' has been addressed by the highest number of challenges. More than 50 challenges elicited in our review address qualityrelated issues, including security, reusability, flexibility, interpretability, and performance. These quality-related issues are emphasized due to the dynamic nature of service-oriented systems. When facing a number of competitive services that deliver similar functionalities, QoS is one of the few factors that a service consumer uses to decide which services to select. Since services are often cross-organizational (which means that the characteristics of hardware and platforms are unpredictable), delivering, measuring, and testing the quality of the provided services are extremely challenging. Furthermore, when business functions are progressively exposed as services, the requirement for QoS turns to be more critical. Therefore, it is not surprising to see that more than onetenth of the elicited SOSE challenges address quality-related issues.

Next to the topic of 'quality' is the topic of 'services', the building blocks of service-oriented systems. The challenges relating to this topic express the properties that a good service is supposed to encompass. Such properties include loosely coupled, self-managed, discoverable, and composable, just to name a few. The requirements of these properties come from the prospect that services are meant to be composed in order to deliver value-added business functions and that this composition can be achieved dynamically. This prospect also explains why the topics of ' $\mathrm{V} \& \mathrm{~V}$ ', 'testing', 'service composition', 'infrastructure', and 'implementation' are all among the top ten hottest SOSE topics.

As a surprise, we did not expect that many SOSE challenges relating to the topic of 'data'. In order to gain a better insight into this topic, we have looked into the primary studies where data-related SOSE challenges were elicited. The first primary study posing data-related SOSE challenge was published in 2003 by Risse et al. [25]. They proposed a research challenge regarding data management for serviceoriented systems, in particular, how to store data to achieve reliability. Few more challenges were posed in successive 
years, mainly about integrated view of data, data visualization, and data mining. Most of the data-related SOSE challenges were posed in [9] in 2007 by Tsai et al., who also pointed out the important issues related to data provenance in service-oriented environments [26]. Due to the link between data provenances and service composition, reliability, security, and integrity of service-oriented systems, Tsai et al. [9] summarized a list of activities in a SOA data life cycle and corresponding techniques needed to support these activities, from which we elicited 18 SOSE challenges. We notice that two-thirds of the data-related SOSE challenges originated there, which means that although many challenges have been elicited, the importance of the topic 'data' has not been commonly recognized in the community. We are reminded that more research efforts could be devoted to data handling in the context of service-oriented environments.

\subsubsection{Topics that are less attractive}

From the bottom of Appendix A, we looked into the topics that appear less attractive to the SOSE community. Surprisingly enough service discovery, a well known SOSE research issue, falls under one of the bottom topics. This unexpected bottom topic triggered us to investigate whether it is true that only two SOSE challenges addressed service discovery. The results of the investigation show that there are in fact a number of challenges addressing other topics that are indirectly related to service discovery. For instance, service description should provide necessary means for service discovery [27](topic: service), federating registries to support service discovery [28](topic: registry), one of the characteristics of services is being discoverable [29](topic: service), infrastructure should be context-aware to find services that are optimal for contexts [30](topic: infrastructure). Each of these challenges focuses on various topics like service, registry, and infrastructure, but overcoming these challenges will eventually contribute to service discovery. These findings reveal that thanks to the active SOSE research efforts of the last years, researchers have gained valuable insight into some SOSE challenges. This insight allows to decompose a too broad challenge into a number of more specific or concrete challenges that often address various topics. In this way, research concerns are better organized and separated, and research directions appear clearer.

\subsubsection{Innovative topics}

We also notice that some topics located at the bottom of Appendix A are quite innovative, for which researchers might have not yet precisely realized the related challenges. For instance, when enterprises start to adopt SOA, and services travel across the organizational boundaries, changes to social and legal aspects are inevitable [31]. These aspects are rela- tively new in the SOSE research community, where researchers usually concentrate on the technology-related issues rather than the social and legal ones.

Moreover, some topics like 'best practice' are typically the interests of practitioners rather than researchers. Since we did not purposely collect publications from the industry, it is reasonable that challenges with respect to SOA practice and application are less frequent in our review, as discussed in Sects. 5.1 and 6.

\subsubsection{The trend of the topics}

Looking at the distribution of the number of topics addressed by the SOSE challenges in each year (see Fig. 6), we notice that it is slightly different from the distribution of the number of the SOSE challenges illustrated in Fig. 4. Instead of the two drops in 2004 and 2006 with regard to the number of SOSE challenges, the number of topics increases each year from 2003 to 2007. Although it drops in 2008, it is still relatively high considering the drop of the number of challenges. In our opinion, the increasing number of topics in each year indicates the fact that the researchers have been keeping identifying new challenges in various SOSE disciplines.

It is also interesting to see that the larger the number of the SOSE challenges is, the longer the corresponding topics exist. Looking at the upper part of Appendix A, we notice that most of the topics are addressed in all the years; while looking at the lower part of Appendix A, we notice that most of the topics are addressed only by the SOSE challenges identified in very recent years. This shows that new SOSE challenges emerge while known challenges remain in the SOSE research community.

More specifically, we observe that all the topics that appear between 2003 and 2006 remain in 2007 and 2008 except for three topics, namely: collaboration, decomposition, and service negotiation. The SOSE challenges that address collaboration point out that business partners of a service-oriented system need to collaborate and work as a team. The SOSE challenges that address decomposition focus on how to decompose an existing application into services. In our opinion, the reason why these two topics disappear after 2006 might be that two broader topics (stakeholder management

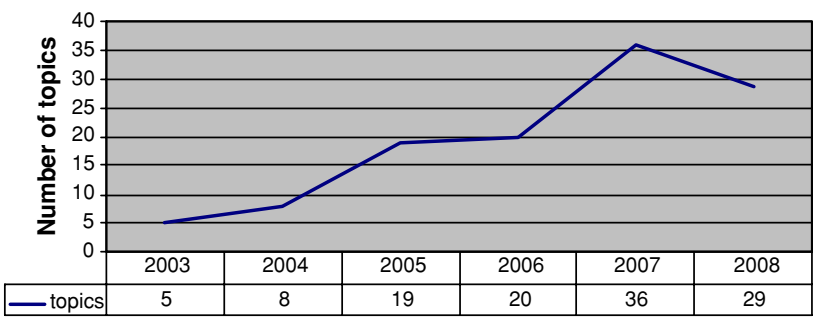

Fig. 6 Distribution of the number of topics by year 
and migration to SOA) attract more attention in recent years. Hence, collaboration and decomposition are replaced by these two broader topics.

It is very surprising to see that only one challenge proposed in 2006 addresses service negotiation. Service negotiation refers to the interaction between a service consumer and provider with the aim of reaching a service level agreement. The automation of this interaction is of great importance for a service-oriented system that dynamically discovers and composes services. Since dynamic service discovery and composition are still under research and are seldom applied in practice, service negotiation does not actively play a role in current service-oriented systems. However, we expect service negotiation to come back as a hotter research topic in near future.

We also observe several topics that emerge in 2008 including service adoption, indicators, social, and legal issues and stakeholder management. These topics are quite innovative and often cross-cut multiple disciplines in SOSE. They concern not only technical issues but also business and organizational issues. The emergence of these topics shows that the scope of SOSE is not limited to IT-related issues; rather, it involves business, stakeholders, organizations, and the society.

\subsection{RQ2.2-types of SOSE challenges}

Following the constant comparison method explained in Sect. 2.3.5, we coded each elicited SOSE challenge and identified a number of distinguishing patterns among the codes. These patterns can be regarded as the properties of a group of SOSE challenges. This confirms our second hypothesisSOSE challenges can be classified based on their types.

\subsubsection{The definition of the eight types of SOSE challenges}

We have identified eight types of SOSE challenges, namely characteristics, quality attributes, artifacts, SOSE activity, service operations, technique, business, , and cross-cutting concerns. Each type of SOSE challenges is described as follows:

Characteristic is an unique feature, peculiarity, requirement or property of services that have been distinguished by the research community. Sometimes, characteristics are also called design principles. Being applicable to all service-oriented systems, characteristics are regarded as non-functional requirements within the scope of systems (indicated as white area in [32]), which means they are of concern for service producers including service developers, service providers, and service integrators. All the service-oriented systems should have the same set of characteristics from the perspective of service orientation. For instance, loose coupling is a characteristic because service producers are supposed to deliver services that have the least dependencies on each other, that are composable with other services and, if changed, the impact is minimized.

Quality attribute refers to the quality or capability that satisfies customer specifications. Different from characteristics, quality attributes are explicitly demanded by service consumers. Consequently, quality attributes often require various disposition on a case-by-case basis (indicated as black area in [32]. For instance, high performance might be important to one service consumer, whereas high security might be the interest of another service consumer.

Artifact is an object or unit that commonly exits in deployed service-oriented systems, such as service, infrastructure, service contract, etc. In this review, we address artifacts from the product point of view. In the other words, artifacts constitute products. Therefore, objects or units not directly used in the deployed systems are not regarded as artifacts here. For instance, requirements specifications are not regarded as artifacts in our review because they are used during the design, development or even the testing phase but not directly involved in the operation of a deployed system. A service specification, however, is an artifact because it is used in service discovery, service composition, and service monitoring, which might occur after services are deployed.

SOSE activity is a task carried out in engineering serviceoriented systems, such as requirement engineering, service design, or testing. The challenges related to SOSE activities are often described in terms of requirements or expectations on these activities. For instance, requirement engineering in SOSE becomes a challenge because system structure, modeling and specification languages, and execution environment all differ from traditional software systems.

Service operation is a special task which is often expected to be automated and executed dynamically in engineering service-oriented systems. We also notice that the objects of such a task are often services. To distinguish these tasks from the SOSE activities that are usually carried out by engineers, we classify this type of task as a service operation.

SOSE technique is a specific method, skill or tool that can be adopted in order to build a system with the required level of quality or encompasses the characteristics of service-oriented systems. A distinctive characteristic of a SOSE technique is that it can often be adopted in different contexts. For instance, simulation is a technique that is often used to simulate new services for evaluating their runtime behavior before deployment. Simulation can also be used in requirements engineering and modeling. However, since this technique is not mature enough to simulate all the (runtime) behavior correctly and completely, research efforts are needed. Once simulation is mature, multiple fields (e.g. requirements engineering, testing) may benefit from it. 
Cross-cutting concern is an issue relevant both to SOSE and to other domains. For instance, SOA education is relevant to both SOSE and the education domain. This type of concerns can be regarded as challenges where research efforts are demanded to leverage issues from diverse domains.

Business-related challenges reflect issues that enterprises have to deal with due to SOA adoption. When enterprises start to expose their business functions as services, they may face challenges related to workload management, business process, stakeholder management, etc. Although these challenges are not directly related to SOSE, business-IT alignment requires the cooperation of the business community and the IT community. Therefore, we also regard business challenges as SOSE challenges.

\subsubsection{Inter-relationship between types of SOSE challenges}

All types of SOSE challenges are potentially inter-related. For instance, SOSE technique challenges can be regarded as a super-type which cross-cuts all the other types. This is because any proposed methods or tools, which are in support of attaining characteristics, quality attributes, required artifacts, engineering tasks, or even aspects relating to business, and other domains, are regarded as technique challenges in our review. As a result, technique challenges are linked to the other seven types of challenges. When a technique challenge is resolved, its inter-related challenges are also benefited.

Another inter-relationship exists between process-related and product-related challenges. Challenges of SOSE activities and service operations are process-related challenges because they concern how to build service-oriented systems that meet the requirements of customers, whereas challenges of characteristics, quality attributes, and artifacts are product-related challenges because they concern what should be built in service-oriented systems to meet the requirements of customers. Since better approaches (how) often lead to better results (what), overcoming process-related challenges are often in support of tackling product-related challenges.

To further investigate the potential inter-relationships between types, we study their distribution over time. In this way we are able to observe the emergence of each type, without entering into the details of the individual challenges belonging to that type (which can be a further issue for future work).

Technique challenges. As presented in Fig. 7, the distribution of the number of SOSE technique challenges is quite similar to that of the other types of SOSE challenges. We observe that publications addressing other types of SOSE challenges often address associated technique challenges. This explains why the two lines in the figure are similar. Nonetheless, this observation is purely based on quantitative aspects; no con-

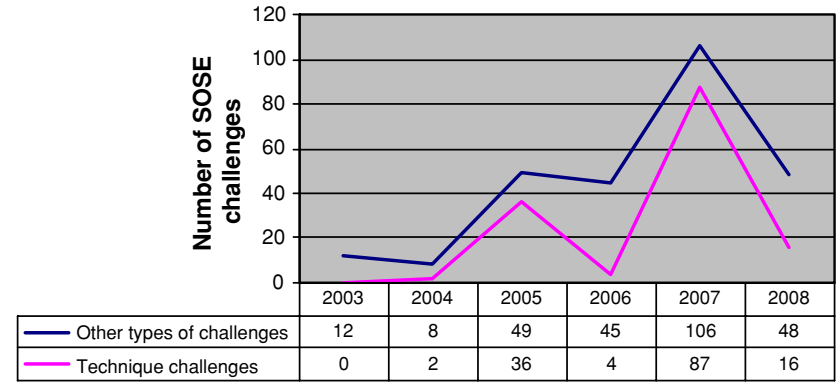

Fig. 7 The distribution of the number of technique SOSE challenges versus other types of SOSE challenges

clusion can be drawn in this review on the extent to which certain technique challenges be indeed inter-related to which other challenges. We will explore inter-relationships between individual challenges in our future work. Furthermore, we observe that the distribution of the number of SOSE technique challenges is also similar to that of the primary studies, which means the average number of technique challenges proposed in each primary study is similar in each year. In our interpretation, this observation shows that SOSE challenges are commonly recognized in the SOSE research community. Product-related SOSE challenges. Further, we studied the distribution of the number of challenges of artifacts, characteristics and $Q o S$, which are the product-related challenges. The graphic in Fig. 8 reveals some interesting facts with regard to the product perspective. First of all, no $Q o S$-related challenges were posed in 2003. This is quite reasonable in that functional requirements can be directly implemented while non-functional requirements often rely on other aspects, such as hardware, network, etc. When the serviceoriented paradigm just emerged, it is logical that less attention is focused on what QoS is demanded from customers, but more on what service-oriented systems should deliver. Second, while the numbers of the other two types of SOSE challenges drop in 2006, the characteristic challenges increased. We believe this is because increasing attention was put on quality and artifacts of service-oriented systems in 2005, researchers were able to elicit their characteristics based on those results. Third, we did not recognize any $Q o S$ and characteristics challenges in 2008 until July, which might indicate that the $Q o S$ challenges have reached their saturation, and focus is being put on engineering solutions.

Process-related SOSE challenges. In addition to these product-related challenges, we also studied the distribution of SOSE activity and service operation challenges, which are the process-related challenges (see Fig. 9). We notice that different from the other seven types of SOSE challenges, the number of SOSE activity challenges does not drop in 2006. Instead, it continuously grows and reaches its peak (35 SOSE activity challenges) in 2007, which is also the highest number as opposed to the other six types (excluding the 


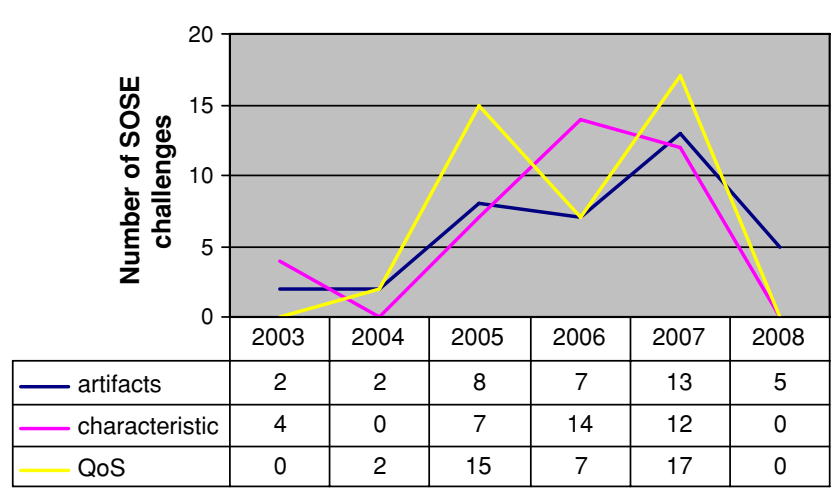

Fig. 8 The distribution of the number of product-related SOSE challenges

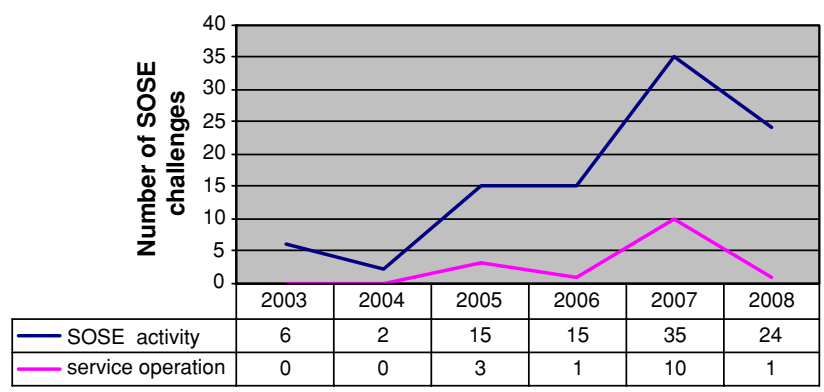

Fig. 9 The distribution of the number of process-related SOSE challenges

super-type: technique challenges) of SOSE challenges proposed each year. The cause of such a high number, in our opinion, is due to the fact that a large number of productrelated SOSE challenges are known by the year of 2007, indicating a set of requirements for service-oriented systems. Consequently, SOSE activity challenges as one type of process-related challenges are proposed in support of these new requirements. Service operation (e.g. service discovery or service composition), another type of process-related challenges, began to play an important role due to the increasing need to provide integrated solutions, which leads to corresponding challenges.

Business-related challenges and cross-cutting concerns. It is surprising to see that business-related challenges and crosscutting concerns start to draw more attention in the research community only in recent years (as depicted in Fig. 10). In our opinion, these challenges should have occurred or been recognized as soon as enterprises started SOA adoption in the beginning of this decade. The reason why business challenges are gaining more attention only recently, in our opinion, is the increasing need for business-IT alignment. As argued by Saugatuck Research [33], SOA adoption happens in three waves, namely project-based, process-based, and programbased. When enterprises just start the journey to serviceorientation, the deployment of services is vertical (i.e. departmental-driven or project-based). In this case, often

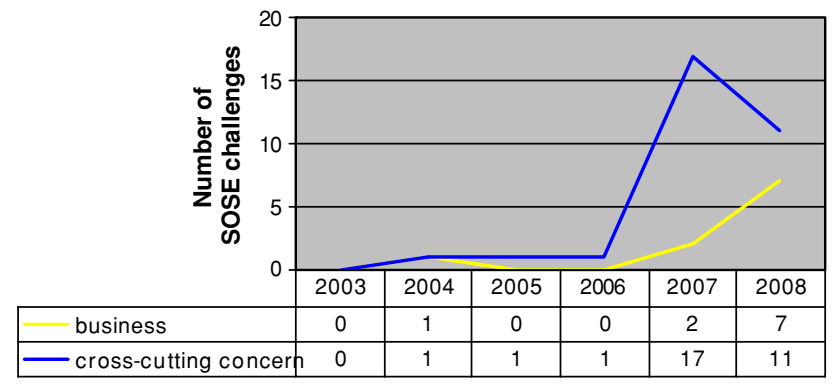

Fig. 10 The distribution of the number of business-related and crosscutting SOSE challenges

legacy systems are wrapped and delivered as services that are used within a department or project. After these pilot projects become mature, enterprises are ready to extend services horizontally (i.e., across-department or process-based). Even at this stage, there are not too many challenges at the business level in that services are created or wrapped on top of legacy systems. Although cooperation between departments might occur, it is still under one business domain and controllable. Until SOA is adopted enterprise-wise (i.e., program-based), which starts recently, the impact of service-orientation on the business level becomes inevitable. Business process models have to be aligned with IT solutions, risk analysis has to be done in the context of SOA, strategic decisions have to be made on which business partners to choose keeping in mind the extent to which the business partners support SOA, just to give few examples. Furthermore, enterprise-wide SOA adoption requires much more time and financial investment, as well as extra concerns relating to governance and security issues, enterprises face many more new challenges.

Next to business-related challenges, enterprise-wide SOA adoption brings more cross-cutting concerns. When SOA has been progressively adopted, challenges related to training, culture, social, and legal issues also emerge. We expect an increasing number of cross-cutting challenges in the future.

\subsection{RQ2.3 - On other ways to classify SOSE challenges}

Instead of classifying the elicited SOSE challenges in terms of the topics that they address and the types they reveal, we found that they can also be classified in terms of the origins or the impacts. For instance, some SOSE challenges emerge due to cultural or organizational factors, and some have impact on projects or management. Based on this idea, we could propose a classification scheme like engineering challenges, project challenges, organizational challenges, cultural challenges, management challenges, etc. However, due to the fact that our primary studies all come from academic computer science publications, most of the elicited SOSE challenges are engineering challenges and only very few challenges could be classified into the other categories. Therefore, we do 
not use this classification scheme in this review. It could be used in future work, however, when we will elicit challenges from industrial SOSE practices, too.

\subsection{The SOSE challenges classified along two dimensions}

In our review, we have identified 51 primary studies where 413 SOSE challenges have been proposed spanning from January 2003 to July 2008. We studied all these SOSE challenges in the context of their corresponding primary studies. By applying data synthesis, we have further determined, for each challenge, its type and covering topic.

The SOSE challenges that address the same topic may have different types. For instance, capturing user requirements and simulation are two SOSE challenges that are both about requirements engineering), where the former is a SOSE activity while the latter is a technique. Similarly, the SOSE challenges that are of the same type may address different topics. For instance, data visualization, runtime ranking mechanism, and dynamic security analysis are three technique challenges which address the topics: data, service selection, and quality, respectively.

All these findings are collectively presented in a cross-referenced table (see Appendix B) showing under each topic, how many challenges have been proposed of each type, or vice versa. This table can be very valuable when researchers need to get an overview of the state-of-the-art research challenges on a specific topic or a specific type of SOSE challenges.

\section{Discussion}

\subsection{Threats to validity}

As an empirical research, the validity of the results from the systematic review have to be evaluated. In particular, due to the fact that subjective measurements have been involved in selecting the primary studies, data extraction and data analysis in the course of the review, the credibility of the results could be argued. Thus, in this section, we justify the validity of the results by discussing possible threats that might restrict our ability to interpret extracted data and conclude our findings.

Suggested by Perry et al. in [34], at least three types of threats should be discussed. This includes (a) threats to construct validity, (b) threats to internal validity, and (c) threats to external validity. Construct validity means to what extent the inferences can be made correctly (i.e., consistent understanding between study designers and executors). Internal validity focuses on the degree to which the study design allows results following data (i.e., bias is eliminated). External validly refers to how far the results of the study can be generalized (i.e., environmental factors do not have impact on findings). In the following we discuss each of them.

Construct validity. In our review, we do not encounter threats to construct validity due to the fact that the review was carried out by the same person who designed the review. Thus, the chance of misinterpretation of theoretical concepts is minimized. As the objective of the review is to explore SOSE challenges, SOSE and challenge are two main concepts in our reviews. In the review protocol, we have explicitly defined SOSE challenges as research issues (challenges) in engineering service-oriented systems (SOSE). Such a definition helps readers to achieve the same interpretations and helps researchers to replicate the review (if needed) in the future.

Internal validity. With respect to the process of the systematic review, the main limitation is that the review is mainly conducted by a Ph.D. candidate rather than multiple researchers. Since the decisions that need to be made in selecting primary studies and synthesizing extracted data are quite subjective, the possibility of bias is increased. In order to minimize the chance of bias we have explicitly defined a review protocol beforehand, which includes research questions, search strategies, study selection strategies with precise inclusion, and exclusion criteria, as well as a number of data synthesis methods. The review protocol has been carefully examined by three colleagues with experience in empirical software engineering as well as systematic reviews. After the review protocol was finalized, the review was executed strictly following the protocol. Furthermore, a secondary researcher has conducted a quality assurance check by evaluating randomly selected data set. Nevertheless, with the involvement of colleagues and a secondary researcher, we can minimize but not fully prevent the subjective influence of the conductor of the systematic review.

With respect to the design of the review, we recognize two threats. First of all, to reduce the number of studies that meet the search criteria into a feasible range, we have created a list of related terms tightly related to the two keywords (SOSE and challenge) and restricted the search in abstracts only. Prior to executing the review, we have evaluated the search strategy against a number of studies known to be relevant. The result shows that all these known studies are retrieved by the search strategy. We therefore have the confidence that the search strategy can be used to find other relevant unknown studies. We, however, are not able to prevent the chance that a publication completely relevant to our review is not selected simply because its abstract does not contain the keywords defined in the search string.

Second, we explicitly excluded publications whose objective is only to present SOSE solutions. Our underlying assumption is that if proposing a challenge is not one of the objectives of a publication, the challenge that might be 
addressed in the study is a known challenge, which means it should be originally proposed in other publications. However, it is possible that a publication actually does propose new SOSE challenges, but this objective is not presented in a clear way. In this case, we might not identify this publication as a primary study and therefore miss the new challenge. To gain more confidence on the completeness of elicited SOSE challenges, we have checked the elicited SOSE challenges against the SOSE challenges that are addressed by the publications where proposing SOSE challenges is not one of their objectives. The results of this check show that we did not overlook any SOSE challenges addressed in those publications.

External validity. The scope of our review is restricted purely to the academic domain. We purposely looked for research challenges proposed in the form of scientific papers that are collected by a number of electronic databases which contain only academic research papers. We would assume that SOSE challenges appearing in the academic domain occur in SOSE practice as well since these challenges are simply issues that need to be solved to engineer service-oriented systems, regardless who poses them. However, since SOSE research challenges presented in other forms than scientific papers were not considered in our review, the completeness of the results might be threatened. Nevertheless, our results show that (as explained in Sect. 4) the trend of SOSE challenges, with regard to the number of SOSE challenges posed each year on each topic and each type, is in accordance with the waves of SOA adoption indicated by Saugatuck Technology [33].

Evaluating to what extent the results of one review also hold in replicated reviews is one way to address both internal and external validity threats [35]. With respect to internal validity, bias could be easily detected when different results are concluded in different replicated reviews; and with respect to external validity, whether results of a review hold only under specific conditions can be determined by analyzing underlying conditions of each replicated review.

Due to the feasibility issues, we did not perform replications in our review. However, given the concrete and detailed review protocol, replications can be conducted as soon as practically possible. Other researchers may also replicate the review based on our protocol or carry out an up-to-date review in the future.

\subsection{Quality assessment}

Differently from the other systematic reviews in software engineering, ours did not appraise the quality of the primary studies per se. In our review, we did not study the nature of SOSE; rather, we are interested in what has been proposed as SOSE challenges. Therefore, we do not enter the merit of the quality of the primary studies.

\section{Conclusions and future works}

To gain insight into the current status of SOSE research issues, we have conducted a systematic review exploring SOSE challenges. This paper presents the results of the systematic review as well as the empirical research method we followed.

The main contributions of this work come from an overview of all the SOSE challenges being recognized in the research community and their classification. According to the results of our review, the elicited SOSE challenges can be classified along two dimensions, namely topics and types. In this way, the SOSE challenges assuming different meanings in different studies are clustered, and the SOSE challenges appearing fictively independent are also aggregated. This means, given a specific topic or type (or both), the corresponding inter-dependencies emerge. Consequently, when research efforts are devoted to overcome one of these SOSE challenges, researchers are able to put this challenge in a context of inter-related research issues rather than focus on them in isolation.

In addition to the classification of the SOSE challenges, we also have a number of interesting findings by studying the number of challenges proposed in each year. SOSE emergence laws, one of the most important findings, point out the relationship between expectations, research work on challenges and solutions. We also expect that more businessrelated challenges and challenges across other domains will emerge in the coming years.

We discussed two unexpected findings. Very few challenges directly addressing service discovery make us realize that there are many more challenges indirectly addressing service discovery. This further indicates the need, and usefulness of exploring the inter-relationships between the SOSE challenges. Our results also discover that data-related SOSE challenges require more attention in the research community.

During the course of the systematic review, we realized the importance of inter-relationships between challenges. The classification of the SOSE challenges in this review only indicates inter-relationships in a broad sense, meaning that interrelationships are reflected between clusters of challenges rather than individual ones. In order to have a better insight into the inter-relationships between the SOSE challenges at the level of individual challenges, we plan to extend the review results in the future by extracting, analyzing, and modeling data regarding inter-relationships elicited from the primary studies.

Furthermore, as explained in Sect. 5.1, we plan to conduct another systematic review targeting at industrial SOSE practices since there might be more or different SOSE challenges discussed, concerning SOSE in practice. In addition, we also plan to look into SOSE-related books, magazines, and reports to have a more complete overview of SOSE challenges. 
Studying solutions to SOSE challenges is another research direction of our future work. After the inter-relationships between the individual SOSE challenges are established and more knowledge about SOSE practice challenges is accumulated, we will be able to study the state-of-the-art in SOSE by defining and using an inter-related SOSE challenge framework.

Acknowledgements The research leading to these results has received funding from the European Community's Seventh Framework Programme FP7/2007-2013 under grant agreement 215483 (S-Cube).
Open Access This article is distributed under the terms of the Creative Commons Attribution Noncommercial License which permits any noncommercial use, distribution, and reproduction in any medium, provided the original author(s) and source are credited.

\section{Appendix A: An overview of the number of SOSE challenges of each topic each year}

See Fig. 11
Fig. 11 An overview of the number of SOSE challenges of each topic each year (sorted by the descending number of challenges under each topic)

\begin{tabular}{|c|c|c|c|c|c|c|c|}
\hline Topics & 2003 & 2004 & 2005 & 2006 & 2007 & 2008 & $\begin{array}{l}\text { Grand } \\
\text { Total }\end{array}$ \\
\hline quality & -- & 2 & 17 & 7 & 27 & -- & 53 \\
\hline service & 3 & 1 & 7 & 12 & 7 & 1 & 31 \\
\hline data & 4 & 1 & 2 & -- & 19 & -- & 26 \\
\hline testing & -- & -- & 11 & 5 & 4 & 5 & 25 \\
\hline verification and validation & -- & -- & 9 & 2 & 11 & 3 & 25 \\
\hline modeling & -- & -- & 4 & -- & 17 & 1 & 22 \\
\hline service composition & -- & -- & 5 & 1 & 11 & 1 & 18 \\
\hline implementation & 2 & -- & 3 & 3 & 4 & 3 & 15 \\
\hline infrastructure & -- & 1 & 6 & -- & 7 & 1 & 15 \\
\hline service specification & 2 & -- & 4 & 3 & 3 & 2 & 14 \\
\hline design & -- & -- & 2 & 1 & 6 & 3 & 12 \\
\hline management & 1 & 1 & 1 & -- & 8 & 1 & 12 \\
\hline transaction & -- & -- & -- & 1 & 11 & -- & 12 \\
\hline BPM-SOA & -- & 1 & 1 & -- & 6 & 2 & 10 \\
\hline governance & -- & -- & -- & -- & 4 & 6 & 10 \\
\hline monitoring & -- & -- & 2 & 1 & 4 & 2 & 9 \\
\hline requirements engineering & -- & -- & -- & 1 & 7 & 1 & 9 \\
\hline evaluation & -- & -- & 4 & -- & 3 & -- & 7 \\
\hline service contracts & -- & -- & -- & 2 & -- & 5 & 7 \\
\hline engineering & -- & -- & -- & -- & 3 & 3 & 6 \\
\hline service provisioning & -- & -- & -- & 1 & 4 & 1 & 6 \\
\hline SOA education & -- & -- & -- & 1 & 2 & 3 & 6 \\
\hline strategy & -- & -- & -- & -- & 2 & 4 & 6 \\
\hline business-IT alignment & -- & -- & -- & -- & 3 & 2 & 5 \\
\hline people & -- & 2 & -- & -- & 3 & -- & 5 \\
\hline development & -- & -- & 3 & -- & 1 & -- & 4 \\
\hline registry & -- & -- & -- & 2 & 1 & 1 & 4 \\
\hline service consumption & -- & -- & -- & 2 & 1 & 1 & 4 \\
\hline fault handling & -- & -- & 1 & -- & 2 & -- & 3 \\
\hline indicators & -- & -- & -- & -- & -- & 3 & 3 \\
\hline maintenance & -- & -- & -- & -- & 1 & 2 & 3 \\
\hline migration to $\mathrm{SOA}$ & -- & -- & -- & -- & 3 & -- & 3 \\
\hline service selection & -- & -- & 2 & -- & -- & 1 & 3 \\
\hline workflow & -- & -- & -- & 1 & -- & 2 & 3 \\
\hline collaboration & -- & -- & 1 & 1 & -- & -- & 2 \\
\hline decomposition & -- & 1 & -- & 1 & -- & -- & 2 \\
\hline laas & -- & -- & -- & -- & 2 & -- & 2 \\
\hline service adoption & -- & -- & -- & -- & -- & 2 & 2 \\
\hline service discovery & -- & -- & -- & -- & 2 & -- & 2 \\
\hline service versioning & -- & -- & -- & -- & 2 & -- & 2 \\
\hline best practice & -- & -- & -- & -- & 1 & -- & 1 \\
\hline service invocation & -- & -- & -- & -- & 1 & -- & 1 \\
\hline service negotiation & -- & -- & -- & 1 & -- & -- & 1 \\
\hline social and legal Issues & -- & -- & -- & -- & -- & 1 & 1 \\
\hline stakeholder management & -- & -- & -- & -- & -- & 1 & 1 \\
\hline Grand Total & 12 & 10 & 85 & 49 & 193 & 64 & 413 \\
\hline
\end{tabular}




\section{Appendix B: An overview of the number of SOSE challenges of each topic and each type}

See Fig. 12

Fig. 12 An overview of the number of SOSE challenges of each topic and each type (sorted in alphabetical order)

\begin{tabular}{|c|c|c|c|c|c|c|c|c|c|}
\hline \multirow[b]{2}{*}{ Topics } & \multicolumn{8}{|c|}{ Types } & \multirow{2}{*}{$\begin{array}{l}\text { Grand } \\
\text { Total }\end{array}$} \\
\hline & business & $\begin{array}{c}\text { service } \\
\text { operation }\end{array}$ & characteristic & $\begin{array}{c}\text { cross-cutting } \\
\text { concern }\end{array}$ & QoS & artifacts & technique & $\begin{array}{l}\text { SOSE } \\
\text { activity }\end{array}$ & \\
\hline best practice & -- & -- & -- & 1 & -- & -- & -- & -- & 1 \\
\hline BPM-SOA & -- & -- & -- & 7 & -- & - & 3 & -- & 10 \\
\hline business-IT alignment & 1 & -- & -- & 3 & -- & -- & 1 & -- & 5 \\
\hline collaboration & -- & -- & 2 & -- & -- & -- & -- & -- & 2 \\
\hline data & -- & -- & -- & -- & -- & -- & 18 & 8 & 26 \\
\hline decomposition & -- & -- & -- & -- & -- & -- & 1 & 1 & 2 \\
\hline design & -- & -- & -- & -- & -- & -- & 6 & 6 & 12 \\
\hline development & -- & -- & 1 & -- & -- & -- & 1 & 2 & 4 \\
\hline engineering & -- & -- & -- & -- & -- & -- & 6 & -- & 6 \\
\hline evaluation & -- & -- & -- & -- & -- & -- & 6 & 1 & 7 \\
\hline fault handling & -- & -- & -- & 1 & -- & -- & 2 & -- & 3 \\
\hline governance & -- & -- & -- & 5 & -- & -- & -- & 5 & 10 \\
\hline laas & -- & -- & -- & -- & -- & -- & 1 & 1 & 2 \\
\hline implementation & -- & -- & 5 & -- & -- & -- & -- & 10 & 15 \\
\hline indicators & 1 & -- & -- & -- & -- & -- & 2 & -- & 3 \\
\hline infrastructure & -- & -- & 2 & -- & -- & 10 & 3 & -- & 15 \\
\hline maintenance & -- & -- & -- & -- & -- & -- & -- & 3 & 3 \\
\hline management & -- & -- & -- & -- & -- & -- & 5 & 7 & 12 \\
\hline migration to SOA & -- & -- & -- & -- & 1 & -- & -- & 2 & 3 \\
\hline modeling & 1 & -- & -- & -- & -- & -- & 20 & 1 & 22 \\
\hline monitoring & -- & -- & -- & -- & -- & -- & 2 & 7 & 9 \\
\hline people & 1 & -- & -- & 4 & -- & -- & -- & -- & 5 \\
\hline quality & -- & -- & 1 & -- & 40 & -- & 12 & -- & 53 \\
\hline registry & -- & -- & -- & -- & -- & 4 & -- & -- & 4 \\
\hline requirements engineering & -- & -- & -- & -- & -- & -- & 6 & 3 & 9 \\
\hline service & -- & -- & 17 & -- & -- & 12 & 2 & -- & 31 \\
\hline service adoption & 1 & -- & -- & 1 & -- & -- & -- & -- & 2 \\
\hline service composition & -- & 10 & -- & -- & -- & -- & 8 & -- & 18 \\
\hline service consumption & -- & -- & -- & -- & -- & -- & -- & 4 & 4 \\
\hline service contracts & -- & -- & -- & -- & -- & 2 & 3 & 2 & 7 \\
\hline service discovery & -- & 2 & -- & -- & -- & -- & -- & -- & 2 \\
\hline service invocation & -- & 1 & -- & -- & -- & -- & -- & -- & 1 \\
\hline service negotiation & -- & -- & -- & -- & -- & -- & -- & 1 & 1 \\
\hline service provisioning & -- & 1 & 1 & -- & -- & - & 2 & 2 & 6 \\
\hline service selection & -- & 1 & -- & -- & -- & -- & 2 & -- & 3 \\
\hline service specification & -- & -- & 1 & -- & -- & 9 & 4 & -- & 14 \\
\hline service versioning & -- & -- & -- & -- & -- & -- & -- & 2 & 2 \\
\hline SOA education & -- & -- & -- & 6 & -- & -- & -- & -- & 6 \\
\hline social and legal Issues & -- & -- & -- & 1 & -- & -- & -- & -- & 1 \\
\hline stakeholder management & -- & -- & -- & 1 & -- & -- & -- & -- & 1 \\
\hline strategy & 5 & -- & -- & 1 & -- & -- & -- & -- & 6 \\
\hline testing & -- & -- & -- & -- & -- & -- & 12 & 13 & 25 \\
\hline transaction & -- & -- & 7 & -- & -- & -- & 3 & 2 & 12 \\
\hline verification and validation & -- & -- & -- & -- & -- & -- & 13 & 12 & 25 \\
\hline workflow & -- & -- & -- & -- & -- & -- & 1 & 2 & 3 \\
\hline Grand Total & 10 & 15 & 37 & 31 & 41 & 37 & 145 & 97 & 413 \\
\hline
\end{tabular}




\section{References}

1. Tsai WT (2005) Service-oriented system engineering: a new paradigm. In: service-oriented system engineering, 2005. SOSE 2005. IEEE International workshop, Beijing, China 3-6

2. Alonso G, Casati F, Kuno H, Machiraju V (2004) Web services: concepts, architectures and applications. Springer, Berlin

3. Voas J, Zhang J (2009) Cloud computing: new wine or just a new bottle? IT Prof 11(2):15-17

4. Turner M, Budgen D, Brereton P (2003) Turning software into a service. Computer 36(10):38-44

5. O'Reilly T (2005) What is web 2.0. http://www.oreilly.com/go/ web2 (30 September 2005)

6. Kontogiannis K, Lewis GA, Smith DB, Litoiu M, Muller H, Schuster S, Stroulia E (2007) The landscape of service-oriented systems: a research perspective. In: International workshop on systems development in SOA environments (SDSOA 2007), IEEE computer society

7. Kontogiannis K, Lewis GA, Smith DB (2008) A research agenda for service-oriented architecture. In: SDSOA 2008: Proceedings of the 2nd international workshop on Systems development in SOA environments, ACM 1-6

8. Papazoglou MP, Traverso P, Dustdar S, Leymann F (2007) Service-oriented computing: state of the art and research challenges. Computer 40(11):38-45

9. Tsai WT, Wei X, Paul R, Chung JY, Huang Q, Chen Y (2007) Service-oriented system engineering (SOSE) and its applications to embedded system development. Service Oriented Computing and Applications 3-17

10. Lpez N, Casallas R, Villalobos J (2007) Challenges in creating environments for soa learning. In: International workshop on systems development in SOA environments (SDSOA)

11. Brereton O, Gold N, Budgen D, Bennett K, Mehandjiev N (2005) Service-based systems: a systematic literature review of issues. Technical report, Keele University

12. Kitchenham B (2007) Guidelines for performing systematic literature reviews in software engineering. Technical report, EBSE Technical Report

13. Khan K, Riet Gt, Glanville J, Sowden A, Kleijnen J (2001) Undertaking systematic review of research on effectiveness. Technical Report CRD report number 4, NHS centre for reviews and dissemination, University of York

14. Zhu H (2005) Challenges to reusable services. In: 2005 IEEE international conference on services computing (SCC 2005), IEEE 243-244

15. Huhns MN, Singh MP (2005) Service-oriented computing: key concepts and principles. IEEE Internet Comput 9(1):75-81

16. Lee SP, Chan LP, Lee EW (2006) Web services implementation methodology for soa application. In: 2006 IEEE international conference on industrial informatics, Singapore, IEEE 335-340

17. Gu Q, Lago P (2007) A model for exploring the service-oriented software engineering (sose) challenges. In: 2nd European young researchers workshop on service oriented computing (YRSOC) $44-49$
18. Dybå T, Dingsøyr T (2008) Strength of evidence in systematic reviews in software engineering. In: ESEM 2008: proceedings of the second ACM-IEEE international symposium on Empirical software engineering and measurement, ACM 178-187

19. Noblit GW, Hare RD (1988) Meta-ethnography: synthesising qualitative studies. Sage, Newbury Park, California

20. Strauss A, Glaser B (1967) The discovery of grounded theory: strategies for qualitative research. Wiedenfeld and Nicholson, London

21. Seaman CB (1999) Qualitative methods in empirical studies of software engineering. IEEE Trans Softw Eng 25(4):557-572

22. Miles MB, Huberman M (1994) Qualitative data analysis: an expanded sourcebook. Sage, Newbury Park

23. Lehman MM, Perry DE (1997) Metrics and laws of software evolution the nineties view. In: Proceedings of the 4th international software metrics symposium (METRICS), IEEE

24. Gartner Inc (2008) Understanding gartner's hype cycles. http:// www.gartner.com/pages/story.php.id.8795.s.8.jsp

25. Risse T, Knezevic P (2003) Data storage requirements for the service oriented computing. In: Applications and the internet workshops, 2003, IEEE 67-72

26. Tsai WT, Zhang XWD, Paul R, Chen Y, Chung JY (2007) A new SOA data-provenance framework. In: Proceedings of the eighth international symposium on autonomous decentralized systems, IEEE computer society $105-112$

27. Papazoglou MP (2003) Service-oriented computing: Concepts, characteristics and directions. In: Proceedings of the fourth international conference on web information systems engineering (WISE), IEEE computer society

28. Blake MB, Huhns MN (2008) Web-scale workflow: integrating distributed services. IEEE Internet Comput 12(1):55-59

29. Tsai WT, Malek M, Chen Y, Bastani F (2006) Perspectives on service-oriented computing and service-oriented system engineering. In: Proceedings of the second IEEE international symposium on service-oriented system engineering (SOSE), IEEE computer society $3-10$

30. Siljee J, Vintges S, Nijhuis J (2005) A context architecture for service-centric systems. In: Location- and context-awareness, vol 3479. Springer, Berlin

31. Contenti M, Termini A, Mecella M, Baldoni R (2003) An e-servicebased framework for inter-administration cooperation. In: Knowledge management in electronic government. Springer, Berlin

32. Bass L, Clements P, Kazman R (2003) Software Architecture in Practice. Addison-Wesley, Reading

33. West M, Guptill B, Koenig M (2006) SOA reality check: three waves of adoption through 2012. Technical report, Saugatuck Technology Inc., USA

34. Perry DE, Porter AA, Votta LG (2000) Empirical studies of software engineering: a roadmap. In: Future of software engineering (ed) Anthony Finkelstein, ACM Press, New York 345-355

35. Shull FJ, Carver JC, Vegas S, Juristo N (2008) The role of replications in empirical software engineering. Empirical Softw Engg 13(2):211-218 